\title{
ENTPD5, an Endoplasmic Reticulum UDPase, Alleviates ER Stress Induced by Protein Overloading in AKT-Activated Cancer Cells
}

\author{
Z. Shen, S. Huang, M. FAnG, AND X. Wang \\ National Institute of Biological Sciences, Beijing 102206, China \\ Correspondence: wangxiaodong@nibs.ac.cn
}

\begin{abstract}
PI3 kinase (PI3K) and tensin homolog (PTEN) lipid phosphatase control the level of cellular phosphatidylinositol $(3,4,5)$-trisphosphate, an activator of AKT kinase that promotes cell growth and survival. Gain-of-function mutations in PI3K and lossof-function mutations in PTEN that activate AKT are commonly observed in human cancers. The activation of AKT causes increased protein translation and the influx of proteins into the endoplasmic reticulum (ER). The expression of ENTPD5, an ER enzyme, is up-regulated in cancer cell lines and primary human tumor samples in which AKT is activated. ENTPD5 hydrolyzes UDP in the ER to promote protein $N$-glycosylation and refolding. The elevation of ENTPD5 activity therefore protects AKT-active cancer cells from protein-overloading-induced ER stress and the resulting growth arrest and apoptosis.
\end{abstract}

Hallmarks of cancer are rapid growth and the proliferation of cancerous cells (Hanahan and Weinberg 2011). Cancer cells often activate distinct metabolic programs to support their rapid growth. One unique phenomenon commonly observed in cancers is called the "Warburg effect," which entails the enhanced metabolism of glucose by aerobic glycolysis in tumor cells.

The phosphoinositide-3 kinases (PI3Ks) and lipid phosphatase tensin homolog (PTEN) regulate cell growth and survival (Yuan and Cantley 2008), and the activation of PI3Ks and the inactivation of PTEN are frequently observed in human cancers (Sun et al. 2009). After the activation of PI3Ks, $\mathrm{PIP}_{2}$ is phosphorylated to form $\mathrm{PIP}_{3}$, which then recruits and activates downstream effectors, such as the Ser/Thr kinase AKT (Whitman et al. 1988; Franke et al. 1997). The activation of AKT in cancer cells accelerates many metabolic programs, such as nutrient uptake and protein synthesis, that accommodate the rapid biosynthesis and growth of cancer cells (Fingar et al. 2002).

Previously, we identified an AKT-up-regulated endoplasmic reticulum (ER)-UDPase, ENTPD5, that links ATP consumption to increased glycolytic influx (Fang et al. 2010). ENTPD5 was purified from PTEN-null mouse embryonic fibroblast (MEF) cells in which increased ATP hydrolysis was observed (depicted in Fig. 1). To our surprise, we found that ENTPD5 itself is a UDP-hydrolysis enzyme and does not directly hydrolyze ATP. The total reconstitution of ATP hydrolysis activity also requires UMP/CMP kinase-1 (CMPK-1), adenylate kinase 1 (AK1), and a small molecule, UMP or UDP, which is present at similar levels in both PTEN wild-type cells and PTEN null cells (Fig. 1A). In vitro, ENTPD5 hydrolyzes two molecules of UDP to form two molecules of UMP. CMPK-1 then phosphorylates the UMP to generate UDP and, in the process, consumes ATP to produce ADP. AK1 then converts two molecules of ADP into one molecule each of ATP and AMP. The entire futile cycle converts one molecule of ATP into AMP (Fig. 1B). In vivo, this cycle links ATP hydrolysis to the protein refolding process in the ER by a series of glucose and phosphate transfer reactions. The UMP generated by ENTPD5 is pumped out of the ER by an antiporter in exchange for UDP-glucose, a building block for the reglucosylation catalyzed by the UDP-glucose:glycoprotein glycotransferase (UGGT). In the cytosol, UMP is phosphorylated by multiple kinases, including CMPK-1 and nucleosidediphosphate kinase (NDPK), to regenerate UDP-glucose. The reglucosylation of misfolded proteins allows for the binding and release of calnexin/calreticulin (CNX/ CRT), two molecular chaperones, to the nascent polypeptide chains until the target proteins are correctly folded (Trombetta and Parodi 2003). The levels of highly glycosylated proteins, especially such tumor-promoting growth factor receptors as EGFR, HER-2, and IGFR- $\beta$, are regulated by ENTPD5 in the ER in this manner. In the reaction catalyzed by UGGT, UDP is generated as a by-product that inhibits the reglucosylation reaction. By hydrolyzing UDP to UMP, ENTPD5 relieves the end-product inhibition of protein reglucosylation and folding in the ER and provides the substrate for UDP-glucose import into the ER by the antiporter (Fig. 1C).

In addition to promoting cell growth and protein folding, ENTPD5 consumes a large amount of ATP through the ENTPD5/CMPK1/AK1 futile cycle. To maintain the intracellular ATP levels in cells containing elevated ENTPD5 levels, cancer cells turn to aerobic glycolysis to produce additional ATP, a process known as the 
A
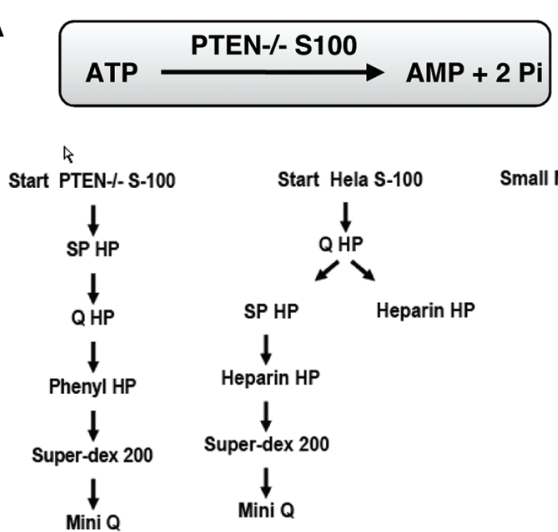

Mini Q

(1)

ENTPD5

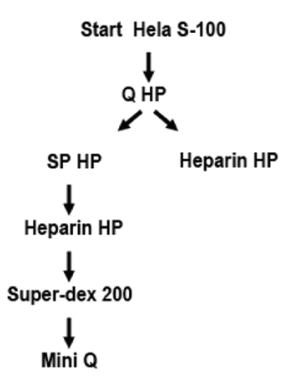

Small Molecule

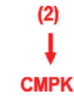

B

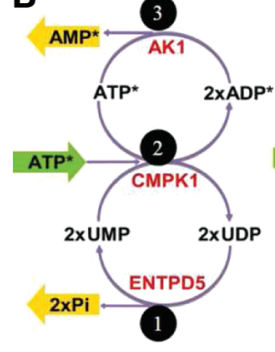

$2 \times A D P^{*} \stackrel{A K 1}{\longrightarrow} A^{A T P^{*}+A M P *}$

$\mathrm{ATP}^{\star}+\mathrm{ATP}^{\star}+2 \mathrm{XUMP} \stackrel{\text { CMPK }}{\longrightarrow} 2 \times \mathrm{UDP}^{\mathrm{N}}+2 \mathrm{XADP} \mathrm{P}^{\star}$ $2 \times U D P \stackrel{\text { ENTPD5 }}{\longrightarrow} 2 \times U M P+2 \times P i$

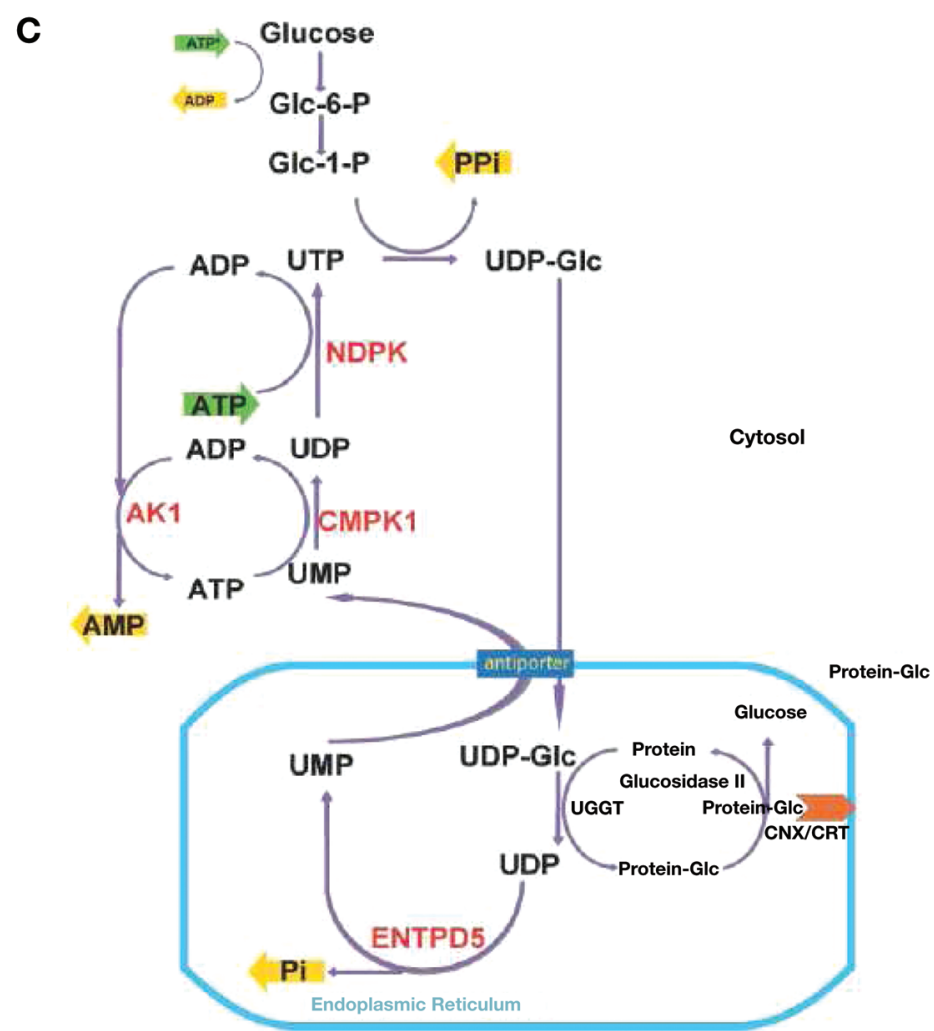

Figure 1. ENTPD5 with CMPK1, AK1, and UDP form a futile cycle to hydrolyze ATP. (A) PTEN null MEF cell has higher ATP hydrolysis activity. Four different components (three proteins and one small molecule) are required to reconstitute the activity. ENTPD5, which is present in PTEN null cells, but not PTEN heterozygous cells, is purified from PTEN null S100 using the ATP hydrolysis assay. CMPK-1 and AK1 are purified from Hela S100. The small molecule is identified as UMP. $(B)$ ENTPD5, AK1 (adenylate kinase), and CMPK-1/UMPK form a cycle to hydrolyze ATP in vitro. In one cycle, ENTPD5 hydrolyzes two molecules of UDP and generates two molecules of Pi and UMP. CMPK-1 converts two molecules of UMP back to UDP and at the same time generates two molecules of ADP. AK1 converts two molecules of ADP to AMP and ATP. The net reaction is hydrolyzing ATP to AMP and two molecules of Pi. $(C)$ ENTPD5 hydrolyzes UDP in the ER to relieve end-product inhibition to reglucosylation enzyme UGGT in vivo. UMP is then exported to cytosol in exchange for UDP-glucose (UDP-Glc). In the cytosol, UMP is converted back to UDP-glucose by a series of phosphatetransfer reactions. In the ER, UDP-glucose is used as a glucose donor to reglucosylate unfolded protein. 
"Warburg effect." ENTPD5 contributes to the "Warburg effect" by promoting higher levels of growth factor receptors at the cell surface and elevating the level of AKT kinase, which then activates downstream glucose transporter activity. In addition, ENTPD5 may also transiently change the intracellular ATP/AMP ratio to allow the allosteric activation of phosphofructokinase (PFK), the key enzyme controlling the influx of glucose into the glycolysis pathway.

\section{Knockdown of ENTPD5 Causes ER Stress-Induced Apoptosis under Protein Overload Conditions}

As reported previously, the knockdown of ENTPD5 in LNCaP cells slowed down cell growth by eliminating growth factor receptors from the cell (Fang et al. 2010). To further characterize the functional significance of ENTPD5 expression in human cancer cells, we used puromycin to induce protein overloading in the ER lumen. Puromycin promotes the dissociation of nascent polypeptides from the ribosome and the translocon, thus increasing the ER load of unfolded proteins (Blobel and Sabatini 1971; Oyadomari et al. 2006). Because ENTPD5 relieves protein-folding stress in the ER, we reasoned that the knockdown of ENTPD5 would make cancer cells more sensitive to increased unfolded protein overloading. As previously reported, we used a Tet-inducible knockdown the LNCaP cancer cell line to test this hypothesis (Fang et al. 2010). In this cell line, the knockdown of ENTPD5 is achieved by adding doxycycline (Dox) to the culture medium. Inducing ENTPD5 knockdown by adding Dox resulted in significant apoptosis, as indicated by the increased cleavage of caspase 3 (Fig. 2A, lane 8), when these LNCaP cells knocked down by ENTPD5 were treated with puromycin for $24 \mathrm{~h}$. Consistent with the increased cleavage of caspase 3 , caspase 3 activity was also increased (Fig. 2B, lane 8).

Regarding the mechanism of cell death, we found that the knockdown of ENTPD5 induced ER stress, as shown by the up-regulated expression of the ER stress markers, $\mathrm{BiP}$ and CHOP, under protein-overload conditions (Fig. 2A, lane 8). We also observed an increase in the spliced form of Xbp1 under such conditions (Fig. 2E, lane 4). After activation of ER stress, activated IRE1 endonuclease removed 26 nucleotides from the unspliced form of Xbp1 messenger RNA (mRNA) (473 bp by reverse transcriptase-polymerase chain reaction, RTPCR) and generated a spliced short form of the mRNA (447 bp by RT-PCR) (Fig. 2E, lane 4) (Yoshida et al. 2001). Additionally, under protein-overload conditions, the knockdown of ENTPD5 led to decreased expression of receptor tyrosine kinases, such as EGFR and HER-2 (Fig. 2A).

To confirm that the above-mentioned cell death and ER stress phenotypes following the knockdown of ENTPD5 were specific, we introduced an ENTPD5 gene with silent mutations in the short hairpin RNA (shRNA) target sequence into the ENTPD5 knockdown LNCaP cells. Although the knockdown of the endogenous ENTPD5 continued after the addition of Dox in these cells (Fig. 2C, lanes 2 and 4), the expression of the shRNA-resistant wild-type transgene (with three FLAG tags fused to the ENTPD5 coding sequence) reversed the increased cleavage of caspase 3 , increased caspase 3 activity, and induction of BiP (Fig. 2C, lane 2). In contrast, the introduction of a catalytic-dead mutant (ENTPD5 CD) that has no UDP-hydrolysis activity was not able to inhibit the cleavage of caspase 3 or the induction of BiP (Fig. 2C, lane 4 and Fig. 2D, lane 4).

\section{EGFR is Degraded by the ER-Associated Degradation (ERAD) Pathway}

The proteins levels of several growth factor receptors, including EGFR and HER-2, were significantly reduced after the induction of protein overloading in ENTPD5 knockdown cells (Fig. 3A), whereas the mRNA levels of EGFR and HER-2 did not decrease, as measured by RT-PCR (Fig. 3B). Therefore, we hypothesized that the above-mentioned decrease in the protein levels of tyrosine kinase receptors was the result of the ER-associated degradation pathway, which involved proteasomal degradation. This hypothesis was supported by the fact that the rapid decrease in the protein level of EGFR was blocked by treating the cells with the proteasome inhibitor MG132 (Fig. 3C, lanes 5 and 6).

To confirm that the degradation of EGFR occurs through the ER-associated degradation pathway, we examined the interaction of ER chaperon proteins with EGFR using a coimmunoprecipitation assay. It is known that monoglucosylated proteins are retained in the ER through interactions with CNX or CRT (Trombetta and Parodi 1992). To determine whether EGFR interacts with these chaperons, we immunoprecipitated the EGFRassociated proteins using an anti-EGFR antibody. Increased interactions between the chaperon proteins CRT/ CNX, BiP, and EGFR were observed when ENTPD5 was knocked down (Fig. 3D, lanes 1 and 2) and when the cells were treated with puromycin (Fig. 3D, lanes 1 and 3). The presence of these chaperons was also increased when the ENTPD5 knockdown cells were treated with puromycin (Fig. 3D, lanes 2 and 4). Taken together, these results demonstrate that aberrantly glycosylated EGFR was unfolded, retained in the ER by CNX/CRT, and then degraded by ERAD when ENTPD5 was knocked down. We thus concluded that ENTPD5 is a crucial component of the ER quality control system for glycosylated proteins, including highly glycosylated growth factor receptors.

\section{DISCUSSION}

\section{ENTPD5 Controls the Folding of Growth Factor Receptors and Promotes Cancer Growth}

Our previous data and the data provided herein highlight ENTPD5 as an important regulatory enzyme in PI3K/AKT-activated cells, in which accelerated protein translation demands higher folding capacity in the ER. 


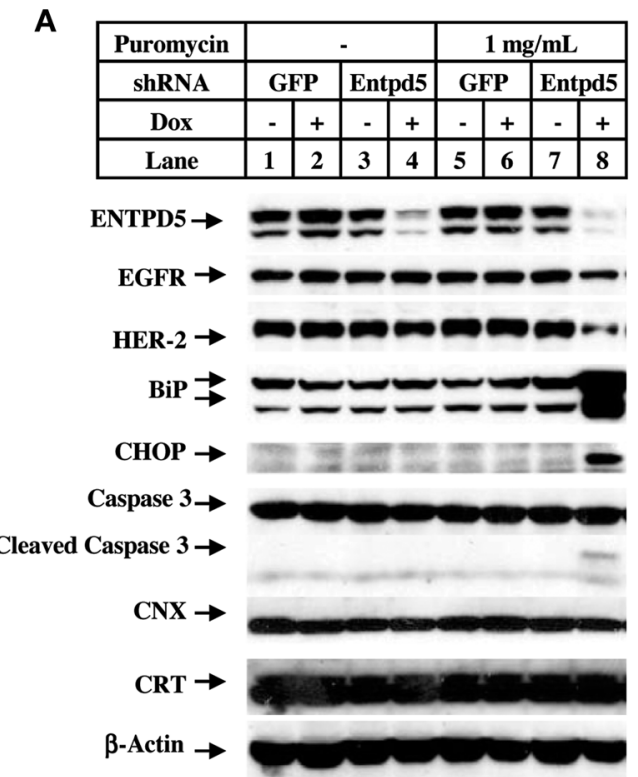

B

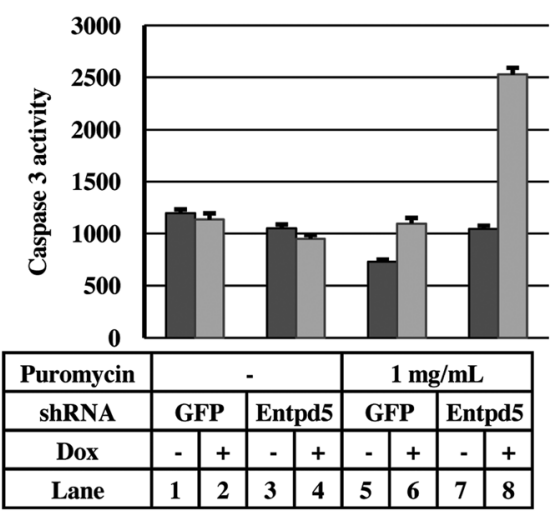

Figure 2. Knockdown of ENTPD5 caused ER stress and apoptosis under protein overload conditions. (A) Knockdown of Entpd5 induced ER stress markers $\mathrm{BiP}$ and $\mathrm{CHOP}$ up-regulation under protein-overloading conditions induced by puromycin. Entpd5 was knocked down by adding doxycycline. Protein overloading was induced by puromycin. Cell lysates were separated by sodium dodecyl sulfate-polyacrylamide gel electrophoresis (SDS-PAGE) and blotted with antibodies as indicated. Tyrosine kinase receptors (EGFR and HER-2) decreased and caspase 3 was cleaved after knockdown of Entpd5. (B) Caspase 3 activity increased after inducing protein overloading in Entpd5 knockdown LNCaP cells but not in green fluorescent protein (GFP) knockdown control cells. Caspase 3 activity was measured using fluorogenic substrate (see the Materials and Methods section below). (C) Overexpression of an shRNA-resistant Entpd5 but not the catalytic-dead form of Entpd5 (Entpd5 CD) attenuated BiP up-regulation and reversed caspase 3 activation and degradation of tyrosine kinase receptors (EGFR and HER-2). (D) Ectopic expression of Entpd5 but not the catalytic-

dead form of Entpd5 (Entpd5 CD) reversed up-regulation of caspase 3 activity under stress condition $(1 \mathrm{mg} / \mathrm{mL}$ puromycin). (E) Knockdown of Entpd5 induced Xbp1 splicing under protein-overloading conditions induced by puromycin. (U) denotes the reverse transcriptase-polymerase chain reaction (RT-PCR) product from unspliced Xbp1 mRNA; (S) denotes the RT-PCR product from spliced Xbp1 mRNA.
C

\begin{tabular}{|c|c|c|c|c|}
\hline cell line & \multicolumn{3}{|c|}{ shRNA Entpd5 } \\
\hline Puromycin & \multicolumn{4}{|c|}{$1 \mathrm{mg} / \mathrm{mL}$} \\
\hline Overexpression & \multicolumn{3}{|c|}{ Entpd5 } & \multicolumn{2}{|c|}{$\begin{array}{c}\text { Cntpd5 } \\
\text { CD }\end{array}$} \\
\hline Dox & - & + & - & + \\
\hline Lane & 1 & 2 & 3 & 4 \\
\hline
\end{tabular}

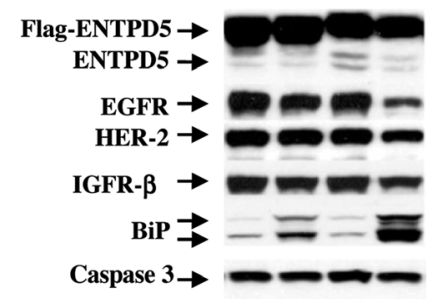

Cleaved Caspase $3 \rightarrow$

$\beta$-Actin $\rightarrow$

D

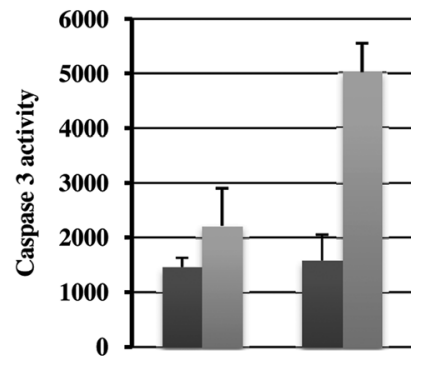

\begin{tabular}{|c|c|c|c|c|}
\hline cell line & \multicolumn{4}{|c|}{ shRNA Entpd5 } \\
\hline Puromycin & \multicolumn{4}{|c|}{$1 \mathrm{mg} / \mathrm{mL}$} \\
\hline Overexpression & Entpd5 & \multicolumn{2}{|c|}{$\begin{array}{c}\text { Cntpd5 } \\
\text { CD }\end{array}$} \\
\hline Dox & - & + & - & + \\
\hline Lane & 1 & 2 & 3 & 4 \\
\hline
\end{tabular}

E

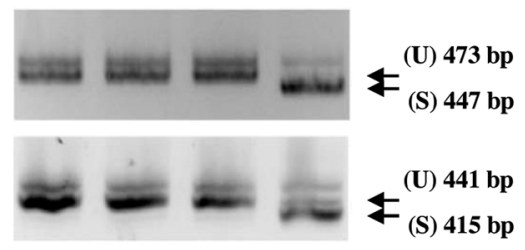

(S) 415 bp

\begin{tabular}{|c|c|c|c|c|}
\hline cell line & \multicolumn{4}{|c|}{ shRNA Entpd5 } \\
\hline Puromycin & \multicolumn{2}{|c|}{-} & \multicolumn{1}{|c|}{$1 \mathrm{mg} / \mathrm{mL}$} \\
\hline Dox & - & + & - & + \\
\hline Lane & 1 & 2 & 3 & 4 \\
\hline
\end{tabular}


A

\begin{tabular}{|c|c|c|c|c|c|c|c|c|}
\hline Time & 0 & & 9 & & 13 & 15 & & 21 \\
\hline Dox & & +1 & \pm & 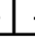 & + & & + & + \\
\hline cell line & \multicolumn{8}{|c|}{ LNCaP shRNA Entpd5 } \\
\hline Lane & 1 & 2 & \begin{tabular}{l|l}
3 & 4 \\
\end{tabular} & & 6 & 7 & 8 & \begin{tabular}{l|l}
9 & 10 \\
\end{tabular} \\
\hline
\end{tabular}

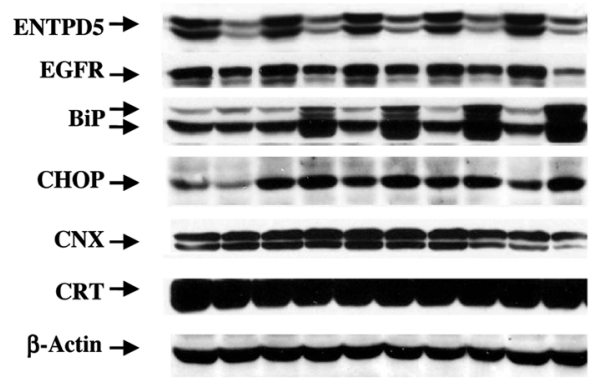

C

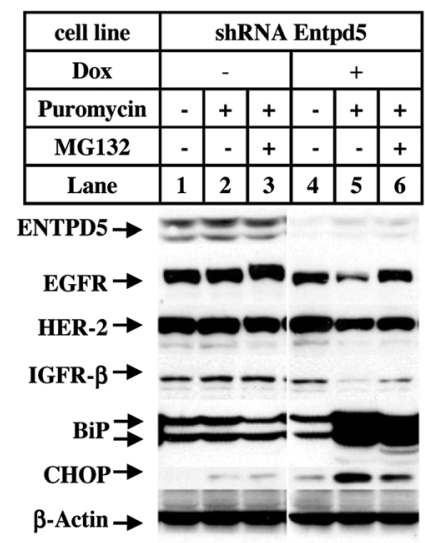

B

\begin{tabular}{|c|c|c|c|c|}
\hline Puromycin & \multicolumn{4}{|c|}{$1 \mathrm{mg} / \mathrm{mL}$} \\
\hline shRNA & \multicolumn{2}{|c|}{ GFP } & \multicolumn{2}{|c|}{ Entpd5 } \\
\hline Dox & - & + & - & + \\
\hline Lane & 1 & 2 & 3 & 4 \\
\hline
\end{tabular}

ENTPD5 $\rightarrow-0$

EGFR $\rightarrow---$

IGFR- $\beta \rightarrow==$

HER-2 $\rightarrow$ -

$\beta$-Actin $\rightarrow-\longrightarrow$

D

\begin{tabular}{|c|c|c|c|c|}
\hline Puromycin & - & - & + & + \\
\hline Dox & - & + & - & + \\
\hline Lane & 1 & 2 & 3 & 4 \\
\hline
\end{tabular}

IP:EGFR $\mid \begin{aligned} & \text { EGFR } \rightarrow-\infty-\infty \\ & \text { CNX } \rightarrow---- \\ & \text { CRT } \rightarrow \text { GE- } \\ & \text { BiP } \rightarrow \text { I }\end{aligned}$

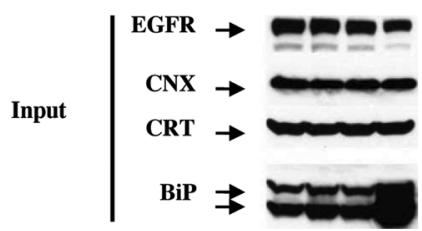

Figure 3. Growth factor receptors are degraded in the ER-associated degradation pathway. $(A)$ Expression level of tyrosine kinase receptors (EGFR and HER-2) decreased rapidly after inducing protein overloading by adding puromycin to Entpd5 knockdown LNCaP cells. $(B)$ mRNA level of receptor kinase receptors after knockdown of Entpd5. mRNA from each treatment was extracted and cDNA was synthesized. EGFR2, HER-2, IGFR, ENTPD5, and $\beta$-actin were amplified from cDNA. The mRNA level of EGFR and IGFR did not decrease after knockdown of Entpd5, and mRNA of HER-2 was slightly decreased after knockdown of Entpd5. (C) Reduction of tyrosine kinase receptors was blocked by proteasome inhibitor MG132. (D) Coimmunoprecipitation of EGFR with BiP, calreticulin (CRT), and calnexin (CNX). Total protein extracts from LNCaP shRNA-Entpd5 cells (without or with doxycycline, Dox) were immunoprecipitated with anti-EGFR antibody and analyzed by western blotting with antibody against ENTPD5, BiP, CNX, and CRT.

In cancer cells, the frequent activation of growth factor receptors and PI3K and the loss of PTEN lead to increased cellular protein translation. Cancer cells therefore need to develop a corresponding system to cope with the pressure from this protein influx. The elevation of ENTPD5 transcription by relieving the inhibition imposed on its promoter by the AKT-regulated FOXO family of transcriptional factors may be one of the mechanisms used by cancer cells (Fang et al. 2010). The elevated level of ENTPD5 hydrolyzes more UDP to yield UMP in the ER, thus relieving the protein-folding pressure and promoting $N$-glycosylation. Therefore, growth factor receptors, such as EGFR and HER-2, are correctly expressed and folded and subsequently maintain the high growth rate of cancer cells. Accordingly, reducing the protein level of ENTPD5 in AKT-activated cells compromised the expression and glycosylation of growth factor receptors and thus may attenuate the growth of cancer cells.

Phylogenetic Studies of UDP-Hydrolyzing Enzymes Indicate the Functional Redundancy of ENTPD Genes

ENTPD5 belongs to a family of ER/Golgi-localized UDP-hydrolyzing enzymes. According to the available literature, there are nine characterized UDP-hydrolyzing enzymes from Saccharomyces cerevisiae, Caenorhabditis elegans, and Homo sapiens. According to their sequence similarities, these proteins can be divided into three classes. Class I (S.Gda1, C.Uda1, and H.Entpd5) and class II (S.Ynd1, C.Mig-23, C.Ntp1, and H.Entpd4) genes are conserved from $S$. cerevisiae to $H$. sapiens, whereas class III genes (C.Apyl and H.Cant1) do not 
have homologs in yeast (Fig. 4. For nomenclature, genus names are followed by gene names). Several of these genes, such as S.Gdal (class I; Abeijon et al. 1993), S.Ynd1 and C.Mig-23 (class II; Gao et al. 1999; Nishiwaki et al. 2004), and C.Apyl (class III; Uccelletti et al. 2008), have been shown to regulate ER stress responses or glycosylation. In humans, there are eight ENTPD family members. ENTPD6 shares the greatest similarity with ENTPD5 and can be categorized as class I; ENTPD7 shares similarity with ENTPD4 and can be categorized as class II. It is possible that all of these genes, including Cant 1, ENTPD4, ENTPD5, ENTPD6, and ENTPD7, have a redundant function in the ER or Golgi. Judging from their localization and enzymatic activity, it would not be surprising if they indeed regulate protein glycosylation under specific conditions. Other ENTPD members (ENTPD1, ENTPD2, ENTPD3, and ENTPD8) are clustered into another class and have been shown to be cytoplasmic membrane or secreted proteins (Wang and Guidotti 1998). These proteins may have distinct functions in regulating nucleotide levels outside of cells.

\section{MATERIALS AND METHODS}

\section{Antibodies}

Antibodies against the following proteins were used in the study: ENTPD5 (Sigma-Aldrich, catalog no. HPA002927), PTEN, AKT, phosphorylated AKT, EGF receptor, Her-2/ErbB-2, IGF receptor $\beta$ subunit, BiP, and $\beta$-actin (Cell Signaling, catalog nos. 9559, 9272, 9271, 2646, 2165, 3027, 3177, and 4970, respectively).

\section{Immunoprecipitation}

Cells were lysed with FLAG lysis buffer (50 mM Tris [pH 7.4], $150 \mathrm{mM} \mathrm{NaCl}$, and 1\% Triton X-100 supplemented with $1 \times$ protease inhibitor cocktail [Roche]). The lysates were cleared by centrifugation at $5000 \mathrm{~g}$ for $5 \mathrm{~min}$, and the supernatant was incubated with antiEGFR-antibody-coupled agarose (sc-03 AC, Santa Cruz) at $4{ }^{\circ} \mathrm{C}$ overnight. The resulting immunoprecipitates were washed three times with lysis buffer, separated on an $8 \%$ SDS-PAGE gel, and analyzed by western blotting using

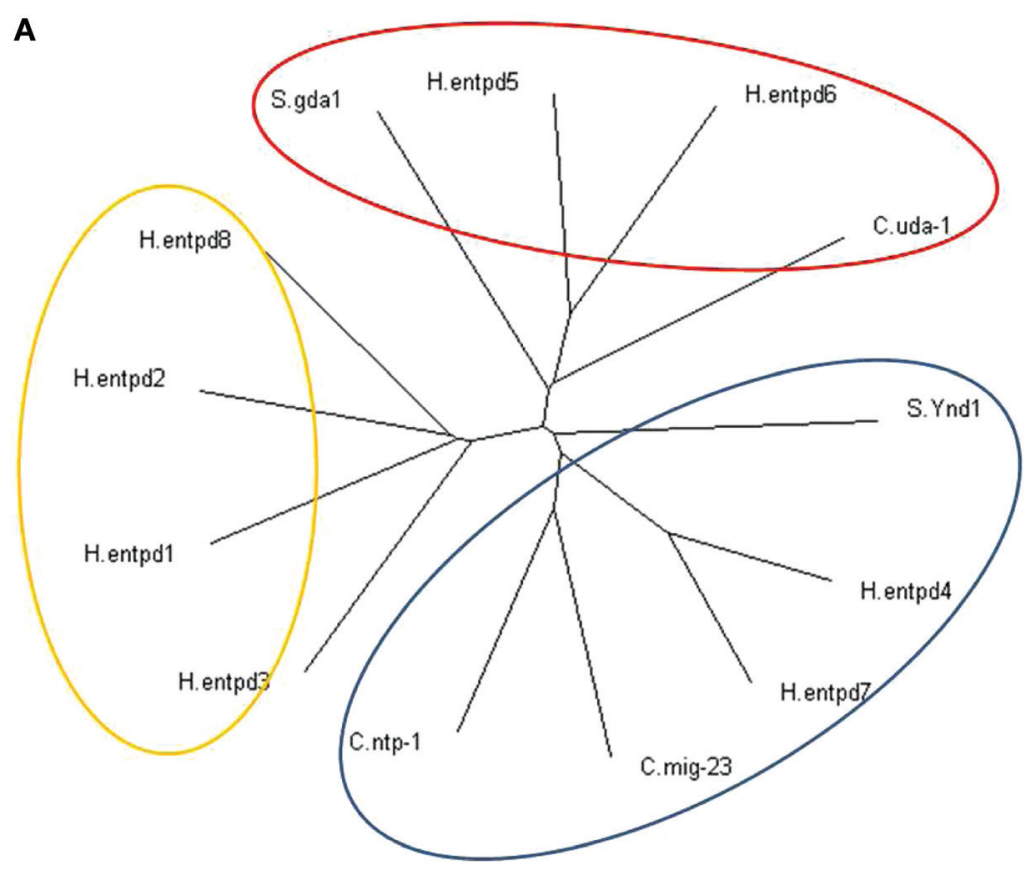

\begin{tabular}{|c|c|c|c|}
\hline B & \multirow{2}{*}{ Gda1 } & \multirow{2}{*}{ Uda1 } & Entpd5 \\
\cline { 3 - 4 } & \multirow{2}{*}{ Ynd1 } & Mig-23 & Entpd6 \\
\cline { 3 - 4 } & & Ntp1 & Entpd7 \\
\hline II & N/A & Apy1 & Cant1 \\
\hline III & S.cerevisiae & C.elegan & H.Sapien \\
\hline Species
\end{tabular}

Figure 4. Phylogenic analysis of Entpd family members from different species. (A) Entpd family members are analyzed using Cluster program, and can be clustered into three different categories according to their sequence similarities. They are conserved from yeast to human. S denotes Saccharomyces cerevisiae. C denotes Caenorhabditis elegans. H denotes Homo sapiens. (B) Gene expansion of ER stress-related apyrases from yeast to human. There are two genes (Gda1 and Ynd1) in S. cerevisiae, four genes (three of them homologs of yeast genes and another one, Apy1, that does not have a homolog in yeast) in C. elegans and five genes (four of them homologs of yeast genes and another, Cant1, a homolog of Apy1 in C. elegans) in H. sapiens. 
Table 1. Primers used for reverse transcription-polymerase chain reaction (RT-PCR)

\begin{tabular}{|c|c|c|}
\hline Gene & RT-PCR primers & Amplicon size (bp) \\
\hline \multirow[t]{2}{*}{$E G F R$} & 5'-TCTCAGCAACATGTCGATGG-3' & 475 \\
\hline & 5'-TCGCACTTCTTACACTTGCG-3' & \\
\hline \multirow[t]{2}{*}{ Her-2 } & $5^{\prime}$-CTGAACTGGTGTATGCAGATTGC-3' & 82 \\
\hline & $5^{\prime}$-TTCCGAGCGGCCAAGTC- $3^{\prime}$ & \\
\hline \multirow[t]{2}{*}{$I G F R$} & $5^{\prime}$-TGGGGAATGGAGTGCTGTAT-3' & 450 \\
\hline & $5^{\prime}$-CGGCCATCTGAATCATCTTG- ${ }^{\prime}$ & \\
\hline \multirow[t]{2}{*}{ ENTPD5 } & 5'-CTTTCCGGAGTGCCTGTTTA-3' & 434 \\
\hline & 5'-AGCCCGTCTCTATGTTGTTCACTT-3' & \\
\hline \multirow[t]{2}{*}{$\beta$-Actin } & $5^{\prime}$-GTGGGGCGCCCCAGGCACCA-3' & 539 \\
\hline & 5'-CTCCTTAATGTCACGCACGATTTC-3' & \\
\hline
\end{tabular}

anti-EGFR (no. 2232 from Cell Signaling or no. sc-03 from Santa Cruz), anti-CRT (no. 2891 from Cell Signaling) or anti-CNX (no. 2433 from Cell Signaling), and antiBiP (Cell Signaling) or antiubiquitin antibodies.

\section{Cell Culture}

$\mathrm{PTEN}^{+/-}$and PTEN ${ }^{-/-}$MEF cells were established as reported previously (Stambolic et al. 1998) and were maintained in Dulbecco's modified Eagle's medium supplemented with 10\% fetal bovine serum (Invitrogen) plus $200 \mathrm{U} / \mathrm{mL}$ penicillin-streptomycin (HyClone). LNCaP-derived stable cell lines were cultured as reported previously (Fang et al. 2010).

\section{RT-PCR}

LNCaP-shRNA-green fluorescent protein (GFP) and LNCaP-shRNA-ENTPD5 cell lines were cultured as described above. After adding Dox for $6 \mathrm{~d}$, the total RNA from each cell line was extracted using TRIzol reagent (Invitrogen). One microgram of total RNA was used for the first-strand cDNA synthesis with superscript RT (Invitrogen). RT-PCR was performed using the primers listed in Table 1 for each gene.

\section{REFERENCES}

Abeijon C, Yanagisawa K, Mandon E, Häusler A, Moremen K, Hirschberg C, Robbins P. 1993. Guanosine diphosphatase is required for protein and sphingolipid glycosylation in the Golgi lumen of Saccharomyces cerevisiae. J Cell Biol 122: 307-323.

Blobel G, Sabatini D. 1971. Dissociation of mammalian polyribosomes into subunits by puromycin. Proc Natl Acad Sci 68: 390-394.

Fang M, Shen Z, Huang S, Zhao L, Chen S, Mak TW, Wang X. 2010. The ER UDPase ENTPD5 promotes protein $N$-glycosylation, the Warburg effect, and proliferation in the PTEN pathway. Cell 143: 711-724.

Fingar DC, Salama S, Tsou C, Harlow E, Blenis J. 2002. Mammalian cell size is controlled by mTOR and its downstream targets S6K1 and 4EBP1/eIF4E. Genes Dev 16: 1472-1487.
Franke TF, Kaplan DR, Cantley LC, Toker A. 1997. Direct regulation of the Akt proto-oncogene product by phosphatidylinositol-3,4-bisphosphate. Science 275: 665-668.

Gao X-D, Kaigorodov V, Jigami Y. 1999. YND1, a homologue of GDA1, encodes membrane-bound apyrase required for Golgi $N$ - and $O$-glycosylation in Saccharomyces cerevisiae. J Biol Chem 274: 21450-21456.

Hanahan D, Weinberg RA. 2011. Hallmarks of cancer: The next generation. Cell 144: 646-674.

Nishiwaki K, Kubota Y, Chigira Y, Roy SK, Suzuki M, Schvarzstein M, Jigami Y, Hisamoto N, Matsumoto K. 2004. An NDPase links ADAM protease glycosylation with organ morphogenesis in C. elegans. Nat Cell Biol 6: 31-37.

Oyadomari S, Yun C, Fisher EA, Kreglinger N, Kreibich G, Oyadomari M, Harding HP, Goodman AG, Harant H, Garrison JL, et al. 2006. Cotranslocational degradation protects the stressed endoplasmic reticulum from protein overload. Cell 126: $727-739$.

Stambolic V, Suzuki A, de la Pompa JL, Brothers GM, Mirtsos C, Sasaki T, Ruland J, Penninger JM, Siderovski DP, Mak TW. 1998. Negative regulation of PKB/Akt-dependent cell survival by the tumor suppressor PTEN. Cell 95: 2939.

Sun X, Huang J, Homma T, Kita D, Klocker H, Schafer G, Boyle P, Ohgaki H. 2009. Genetic alterations in the PI3K pathway in prostate cancer. Anticancer Res 29: 1739-1743.

Trombetta SE, Parodi AJ. 1992. Purification to apparent homogeneity and partial characterization of rat liver UDP-glucose: glycoprotein glucosyltransferase. J Biol Chem 267: 92369240 .

Trombetta ES, Parodi AJ. 2003. Quality control and protein folding in the secretory pathway. Annu Rev Cell Dev Biol 19: 649676.

Uccelletti D, Pascoli A, Farina F, Alberti A, Mancini P, Hirschberg CB, Palleschi C. 2008. APY-1, a novel Caenorhabditis elegans apyrase involved in unfolded protein response signalling and stress responses. Mol Biol Cell 19: 1337-1345.

Wang T-F, Guidotti G. 1998. Golgi localization and functional expression of human uridine diphosphatase. J Biol Chem 273: 11392-11399.

Whitman M, Downes CP, Keeler M, Keller T, Cantley L. 1988. Type phosphatidylinositol kinase makes a novel inositol phospholipid, phosphatidylinositol-3 phosphate. Nature 332: 644-646.

Yoshida H, Matsui T, Yamamoto A, Okada T, Mori K. 2001. XBP1 mRNA is induced by ATF6 and spliced by IRE1 in response to ER stress to produce a highly active transcription factor. Cell 107: 881-891.

Yuan TL, Cantley LC. 2008. PI3K pathway alterations in cancer: Variations on a theme. Oncogene 27: 5497-5510. 


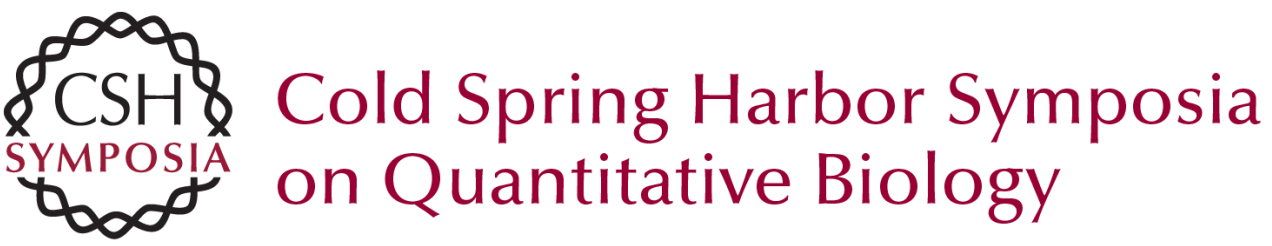

\title{
ENTPD5, an Endoplasmic Reticulum UDPase, Alleviates ER Stress Induced by Protein Overloading in AKT-Activated Cancer Cells
}

\author{
Z. Shen, S. Huang, M. Fang, et al.
}

Cold Spring Harb Symp Quant Biol 2011 76: 217-223 originally published online December 14, 2011

Access the most recent version at doi:10.1101/sqb.2011.76.010876

References This article cites 18 articles, 9 of which can be accessed free at:

http://symposium.cshlp.org/content/76/217.full.html\#ref-list-1

License

Email Alerting
Service $\quad \begin{aligned} & \text { Receive free email alerts when new articles cite this article - sign up in } \\ & \text { the box at the top right corner of the article or click here. }\end{aligned}$ 